\title{
Gastrointestinal cancer surgery and enhanced recovery after surgery (ERAS) during COVID-19 outbreak
}

\author{
G. S. Sica ${ }^{1}$. Michela Campanelli ${ }^{1}$ (D) $\cdot$ V. Bellato ${ }^{1} \cdot$ G. Monteleone $^{2}$
}

Received: 31 March 2020 / Accepted: 15 April 2020/Published online: 11 May 2020

(C) Springer-Verlag GmbH Germany, part of Springer Nature 2020

The outbreak of the coronavirus disease 2019 (COVID-19), first reported in China in December 2019, has become a public health emergency of international concern that also affects patients with digestive diseases $[1,2]$. The Italian government has implemented extraordinary measures to contain the viral spread. Recently, the University Hospital of Rome Tor Vergata has been named "Covid Hospital-4," the fourth referral center of Rome for COVID-19 positive patients (COV-pos). As a result, all the surgical services of the gastrointestinal surgical department, with the exception of one weekly outpatient clinic and one endoscopic session for oncologic and urgent cases, have been locked down. Nonetheless, the emergency department continues admitting surgical patients, even though currently the number of admissions seems to be reduced. The impacts of this situation on both surgical service provision and changes to standard surgical practice are unknown. We share some observations of our initial experience of GI surgery with particular attention to the enhanced recovery after surgery (ERAS) program, in a COVID-Hospital.

Since the lockdown (4 weeks), sixteen patients with known GI malignancies were scheduled to have surgery in the minimally invasive unit of the Tor Vergata Hospital: 8 colonic, 2 rectal, 2 rectal recurrence, and 4 gastric. Furthermore, during the same period, 4 patients required emergency GI surgery.

From referral to preoperative work-up, prehospitalization, admission, and in-hospital care, the whole system had to be adapted to the new COVID-19 hospital setting. However, we have noticed that, since the COVID-19 crisis, our ERAS program is running smoothly and is more effective than ever.

Michela Campanelli

michelacampanelli@live.it

1 Department of Surgical Science, University Hospital Tor Vergata, Viale Oxford 81 00133, Rome, Italy

2 Department of Digestive Disease, University Hospital Tor Vergata, Rome, Italy
Since November 2016, an ERAS protocol is in place and is proposed to all our GI surgical patients. Eighty-two percent of our GI surgery is done laparoscopically, and all patients receive instructions for 5 days of preoperative immunenutritional support. However, given the location of the hospital on the outskirts of the city and it being a referral center for digestive disease, around half of our patients come from small towns and villages with a low level of education, resulting in low penetration of some of the ERAS pillars. More precisely, an estimated $30 \%$ of our patients eventually refuse to leave the hospital prior to an extended period of convalescence. However, since the introduction of the ERAS protocol, we observed an average reduction in the length of stay of 3.5 days (from 8.6 to 5.1 days). Currently, patients seen during the preassessment clinic are not given the prescription for preoperative nutritional support because of changes in the logistics due to restriction in people's motility. Nevertheless, patients appear to understand very well the protocol of enhanced recovery, and in these last few weeks, our median hospital stay was reduced to 4.1 postoperative days. No major complications were observed during our first month of COVID-19 seclusion, and three patients who had a colonic resection left the hospital on postoperative day two: this certainly has already been proven to be both safe and feasible [3] but is not representative of our standard of care. We believe that due to the awareness of SARS-CoV-2 infection, both patients and caregivers had an increased consciousness of the shortage of resources and showed a different attitude toward fast-track discharge.

A strict adherence to the ERAS protocol may be effective in reducing hospital bed shortages and postsurgical associated co-morbidities.

A further consideration regarding the ERAS protocols during the COVID-19 pandemic is bound to its use in laparoscopic surgery [4]. We do not routinely test surgical patients in search of the SARS-CoV-2 infection. Since the beginning of this crisis, some concerns have been raised in regard to operating laparoscopically, especially on patients coming from the emergency department, due to the impossibility of a 


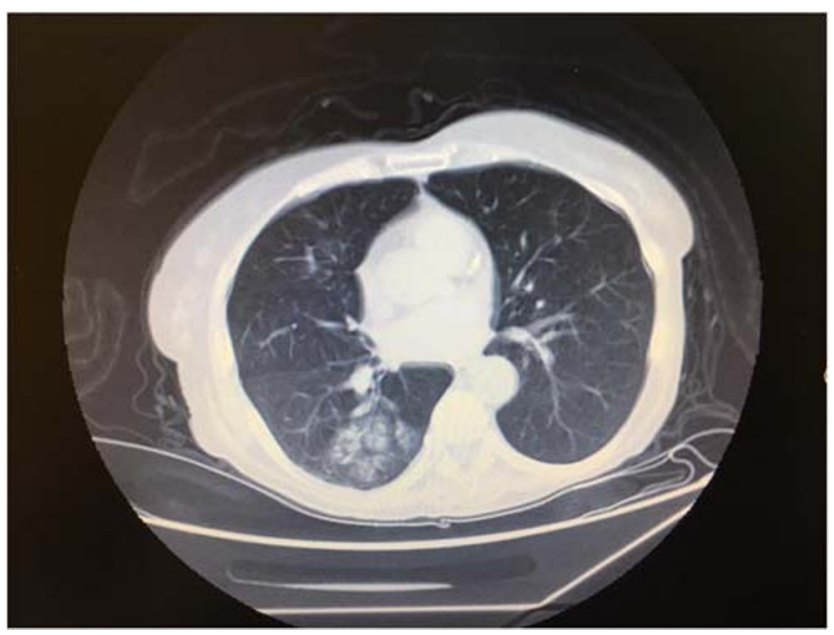

Fig. 1 Chest CT scan: interstitial pneumonia

preoperative screening even in suspected cases. This problem has recently been highlighted [5], and the aerosol generated by the energy devices is certainly amplified by the pneumoperitoneum. However, the risk should remain very low for it is a virus that attacks the respiratory apparatus and is present in the abdominal organs only through the bloodstream. Furthermore, the viral load virus, which has not yet been measured, is probably very low. As a matter of fact, the four patients we admitted for emergency surgery did indeed undergo open surgery. One patient (female, 64 years old) underwent an open Hartmann's procedure for acute perforated diverticulitis. On admission she had an abdominal CT scan but also a chest tomography as part of the new protocol in place for emergency surgical abdominal cases. Given the pictures of her chest CT scan (Fig. 1) and low lymphocytic count, she was treated following the safety protocols for Cov-pos patients. She had two consecutive sets of swabs, both of which negative, in search of the SARS-CoV-2 infection. She made an uneventful surgical recovery and was transferred to respiratory medicine.

Since then, after the discussion with the department of safety in the workplace, specific devices developed to aspirate the smog derived from the use of these energy devices, namely, ConMed Buffalo filters (ConMed Corp. Utice, N.Y., USA), are employed in open and laparoscopic surgery.

The ERAS protocol does not seem to be affected by the COVID-19 crisis. If anything, the crisis has shown once again its potential benefits.
Authors' contributions Sica GS, study conception and design, drafting the manuscript, and critical revision of manuscript. Campanelli M, study conception and design, analysis and interpretation of the data, drafting the manuscript, and critical revision of manuscript. Bellato $\mathrm{V}$, acquisition of data. Monteleone G, critical revision of manuscript. All Authors have contributed and approved this paper.

Availability of data and material The manuscript has associated data in a data repository.

\section{Compliance with ethical standards}

Conflicts of interest The authors declare they have no conflict of interest.

Ethical approval All procedures performed in studies involving human participants were in accordance with the ethical standards of the institutional and/or national research committee and with the 1964 Helsinki Declaration and its later amendments or comparable ethical standards. This article does not contain any studies with animals performed by any of the authors.

Informed consent Informed consent was obtained from all individual participants included in the study.

\section{References}

1. Wu Z, McGoogan JM (2020) Characteristics of and important lessons from the coronavirus disease 2019 (COVID-19) Outbreak in China. Summary of a Report of 72314 Cases From the Chinese Center for Disease Control and Prevention. JAMA 2020:25-28

2. Mao R, Liang J, Shen J, Gosh S, Zhu L-R, Yang H, Wu K-C, Chen M-H (2020) Implication of COVID-19 for patients with pre-existing digestive disease. Lancet Gastroenterol Hepatol 5:426-428

3. Zeng MH, Boni L, Fingerhut A (2020) Minimally Invasive Surgery and the Novel Coronavirus Outbreak: Lessons Learned in China and Italy. Ann Surg: Mar 26

4. The Italian ColoRectal Anastomotic Leakage (iCral) study group Colorectal surgery in Italy during the COVI-19 outbreak: a survery from the iCral study group. Updates Surg in press. https://doi.org/10. 1007/s13304-020-00760-3

5. Gignoux B, Gosgnach M, Lanz T, Vulliez A, Blanchet MC, Frering V, Faucheron JL, Chasserant P (2019) Short term outcome of ambulatory colectomy for 157 consecutive patients. Ann Surg 270(2): $317-321$

Publisher's note Springer Nature remains neutral with regard to jurisdictional claims in published maps and institutional affiliations. 